\title{
Preseptal and Orbital Cellulitis: Thirteen Years of Experience and a Novel Scoring System (SNIPPED Score) for Differentiation
}

\section{Rumeysa Yalçınkaya ( $\nabla$ rumeysa_ra@hotmail.com )}

Dr Sami Ulus Gynecology Obstetrics and Child Health and Diseases Training and Research Hospital: SBU Ankara Dr Sami Ulus Kadin Dogum Cocuk Sagligi ve Hastaliklari Egitim ve Arastirma Hastanesi https://orcid.org/0000-0002-0083-0587

Gönül Tanır

Dr Sami Ulus Gynecology Obstetrics and Child Health and Diseases Training and Research Hospital: SBU Ankara Dr Sami Ulus Kadin Dogum Cocuk Sagligi ve Hastaliklari Egitim ve Arastirma Hastanesi

\section{Suna Özdem}

Dr Sami Ulus Gynecology Obstetrics and Child Health and Diseases Training and Research Hospital: SBU Ankara Dr Sami Ulus Kadin Dogum Cocuk Sagligi ve Hastaliklari Egitim ve Arastirma Hastanesi

Meltem Polat

Dr Sami Ulus Gynecology Obstetrics and Child Health and Diseases Training and Research Hospital: SBU Ankara Dr Sami Ulus Kadin Dogum Cocuk Sagligi ve Hastaliklari Egitim ve Arastirma Hastanesi

\section{Zeynep Savaş Şen}

Dr Sami Ulus Gynecology Obstetrics and Child Health and Diseases Training and Research Hospital: SBU Ankara Dr Sami Ulus Kadin Dogum Cocuk Sagligi ve Hastaliklari Egitim ve Arastirma Hastanesi

Rüveyda Gümüşer Cinni

Dr Sami Ulus Gynecology Obstetrics and Child Health and Diseases Training and Research Hospital: SBU Ankara Dr Sami Ulus Kadin Dogum Cocuk Sagligi ve Hastaliklari Egitim ve Arastirma Hastanesi

\section{Aslıhan Yüce Sezen}

Dr Sami Ulus Gynecology Obstetrics and Child Health and Diseases Training and Research Hospital: SBU Ankara Dr Sami Ulus Kadin Dogum Cocuk Sagligi ve Hastaliklari Egitim ve Arastirma Hastanesi

\section{Fatma Nur Öz}

Dr Sami Ulus Gynecology Obstetrics and Child Health and Diseases Training and Research Hospital: SBU Ankara Dr Sami Ulus Kadin Dogum Cocuk Sagligi ve Hastaliklari Egitim ve Arastirma Hastanesi

\section{Research Article}

Keywords: neutrophil-to-lymphocyte ratio, orbital diseases, proptosis, risk scores, sinusitis

Posted Date: August 18th, 2021

DOI: https://doi.org/10.21203/rs.3.rs-792383/v1

License: (c) (i) This work is licensed under a Creative Commons Attribution 4.0 International License. Read Full License 


\section{Abstract}

We aimed to evaluate clinical and laboratory characteristics of children with preseptal cellulitis (PC) or orbital cellulitis (OC) and to determine whether easilyaccessible parameters could be used to predict OC. The data of children diagnosed with PC or OC between January 2008 and December 2020 were evaluated. Patients aged between 1 month and 18 years who were treated with intravenous antibiotics were included. Logistic regression analysis was performed to identify possible parameters in differentiating between PC and OC. A beta coefficient-based method was used to derive the scoring system. A total of 375 patients [202 (53.9\%) boys], of whom 35 (9.3\%) had OC, were evaluated. Median age was 44 (min-max: 1-192) months. Compared to those with PC, patients with OC were older $(p=0.001)$, had fever and upper respiratory tract infection (URTI) symptoms more frequently, and demonstrated prolonged symptom and hospitalization times ( $\mathrm{p} \otimes 0.001$ for all). Significant differences between groups were observed for numerous parameters; however, logistic regression analysis revealed only five parameters independently associated with OC. The SNIPPED score variables and weights were as follows: sinusitis (2 points), neutrophil-tolymphocyte ratio $>6.78$ ( 3 points), platelet count $>420.5 \times 10^{3} / \mathrm{mm}^{3}$ ( 2 points), proptosis ( 4 points) and duration of symptoms $\geq 4$ days ( 4 points). A cut-off of $\geq 7$ points for OC diagnosis was found to have $91.4 \%$ sensitivity, $96.2 \%$ specificity, $71.1 \%$ PPV, $99.1 \%$ NPV and $95.7 \%$ accuracy.

Conclusion: In addition to showing previously known properties of OC versus PC, our study demonstrated that easily-accessible parameters could be used for the identification of OC.

\section{What Is Known}

- Orbital infections are common in the pediatric age group. Although preseptal cellulitis (PC) is benign, orbital cellulitis (OC) may cause complications which may be serious and fatal.

- The definite diagnosis of $\mathrm{OC}$ is via imaging which may neither be readily available nor easy to access in resource limited settings.

\section{What is New}

- A novel SNIPPED score [sinusitis ( 2 points), neutrophil-to-lymphocyte ratio $>6.78$ (3 points), platelet count $>420.5 \times 10^{3} / \mathrm{mm}^{3}$ ( 2 points), proptosis (4 points) and duration of symptoms $\geq 4$ days (4 points)] may be used to identify $\mathrm{OC}$ in pediatric patients.

- $\geq 7$ points from the SNIPPED score was found to have $91.4 \%$ sensitivity, $96.2 \%$ specificity, $71.1 \%$ PPV, $99.1 \%$ NPV and $95.7 \%$ accuracy to distinguish OC from PC.

\section{Introduction}

Orbital infections may be seen in all age groups, but greater frequency is observed in the pediatric population [1]. In cases where the infection penetrates the orbital septum (which forms a barrier between preseptal and post-septal spaces) the condition is called orbital cellulitis (OC). Orbital infections may occur due to spread from the sinuses and by organisms that originate in the upper respiratory tract or skin. Other inciting factors include insect bites, odontogenic infections and trauma [2]. Although preseptal cellulitis (PC) is relatively benign, OC may cause complications including loss of visual acuity, cavernous sinus thrombosis, meningitis, intracranial abscess and septic embolus, if not managed early [3]. Differentiation between PC and OC may be difficult clinically, and there are no laboratory parameters that can directly aid in the discrimination of these two situations.

The aim of this study was to evaluate demographic, clinical and laboratory features, imaging studies, treatment modalities and outcomes of children with orbital infections. Also, we aimed to identify any laboratory parameters that could help differentiate OC from PC, and whether a scoring system could be devised with these parameters.

\section{Patients And Methods}

This retrospective study was conducted by evaluating the data of pediatric patients diagnosed with PC or OC between January 2008 and December 2020 at the Pediatric Infectious Diseases Department of our hospital. A medical database search was made with the following ICD-10 (International Classification of Diseases-10) codes: inflammation of the eyelids (H01), disorders of eyelids (H02.8, H02.9), ocular involvement in infectious diseases (H03.1), and orbital disorders including cellulitis and abscess (H05). Patients aged between 1 month and 18 years who were admitted to the hospital and treated with intravenous (IV) antibiotics were included in the study. Patients who were admitted with symptoms similar to PC with different final diagnosis and patients followed with outpatient oral antibiotic therapy were excluded. The demographic characteristics of patients, symptoms at admission, duration of oral antibiotic therapy before admission, affected eye (unilateral/bilateral and right/left), the duration of symptoms and treatment, the type (preseptal/orbital) and etiological cause (trauma, insect bite, sinusitis, conjunctivitis, odontogenic infections, dacryocystitis) of orbital infection were recorded from patient history and medical files. Laboratory parameters including complete blood count [white blood cell count (WBC), absolute neutrophil count (ANC), absolute lymphocyte count (ALC), and platelet count, mean platelet volume (MPV)], acute phase reactants [C- reactive protein (CRP) and erythrocyte sedimentation rate (ESR)], and culture results (blood, cerebrospinal fluid, and conjunctival swab cultures) were also recorded. Hematological indices, such as neutrophil-to-lymphocyte ratio (NLR), MPV-to lymphocyte ratio (MPVLR), platelet-to-lymphocyte ratio (PLR), were calculated. Finally, indications and findings of imaging studies [orbital computerized tomography (CT), orbital magnetic resonance imaging (MRI)] and whether surgical intervention was performed were recorded from the electronic medical database.

The clinical diagnosis of PC was made according to physical examination findings when there was just eyelid edema with warmth and tenderness without restriction of ocular movements, proptosis or loss of vision. If there were findings of proptosis, limitations in ocular motility with or without additional signs (such as conjunctival inflammation with chemosis, orbital pain, loss of vision and afferent pupillary defect), OC was diagnosed in a preliminary fashion and 
was confirmed according to radiological evidence showing involvement of the orbital soft tissue posterior to the orbital septum [4]. Blood culture had been obtained from all patients. Lumbar puncture (LP) had been performed in infants who had signs of systemic infection and when meningitis could not be excluded.

Ophthalmology and otorhinolaryngology consultations were requested when needed. Imaging studies had been ordered when there was a suspicion of OC or when patients had proptosis, ophthalmoplegia or when physicians had been unable to open patients' eyelids due to extreme eyelid edema (preventing evaluation of ocular movements). If patients needed orbital surgery, they were referred to an ophthalmology department. Beta-lactam/beta-lactamase inhibitors had been commenced empirically in all patients, but treatments were adjusted or changed with respect to culture, antibiotic sensitivity and imaging results. When clinical and laboratory improvement had been achieved, patients were discharged for outpatient follow-up by switching to alternant oral antibiotic therapy. Total duration of treatment (IV plus oral) was at least 10 days in PC and 14 to 21 days in OC.

\section{Statistical Methods}

The statistical analyses of this study were performed via the SPSS software (version 22.0) for the Windows operating system. Normality of distribution in continuous variables was assessed with Q-Q plots and the Shapiro-Wilk test. Given the presence of normal distribution, comparisons between groups were performed with the Student's $t$-test; whereas, the Mann-Whitney Utest was used to compare variables without normal distribution. The distributions of categorical data in the two groups were compared with Chi-squared tests. Multivariate regression via the backward conditional method was performed to determine factors that were independently effective on OC diagnosis. All parameters that demonstrated significant difference between patients with PC and $\mathrm{OC}$ in univariate analysis were included in the model, as well as parameters that were known risk factors of OC (ophthalmoplegia and proptosis). Receiver operating characteristic (ROC) analyses were performed at two stages: the first was to identify cut-off values for continuous variables showing significance in multivariable analysis (for categorical transformation), the second was to identify cut-off values for the scoring system (detailed below). Any $p$ value lower or equal to 0.05 was accepted to demonstrate statistical significance.

\section{Deriving The Scoring System}

As described above, we identified factors that were independently associated with OC diagnosis by performing logistic regression. ROC curves were created for parameters that were found to be significant in multivariable logistic regression and cut-off values were identified with the Youden $J$ method. Next, in order to be able to derive a score system based on categorical factors, we dichotomized continuous variables with cut-off values obtained from initial ROC analyses. A second logistic regression analysis for OC diagnosis was conducted via the enter method with the dichotomous variables identified in the previous step.

Weights were assigned to each significant parameter by rounding the beta coefficients of regression analysis (not odds ratio) to the nearest integer [5]. Finally, scores were calculated for each patient, ROC analysis was performed, and two relevant cut-off points for the score were identified. Sensitivity, specificity, positive predictive value (PPV), negative predictive value (NPV), and accuracy were calculated.

\section{Results}

There were a total of 375 patients of whom 202 (53.9\%) were boys. Thirty-five (9.3\%) children had OC and 340 (90.7 \%) had PC. Overall median age was 44 (min-max: 1-192) months. Median age of patients with OC was higher than the patients with PC (pख0.001).

Symptoms were similar in the PC and OC groups, except for fever and upper respiratory tract infection (URTI) symptoms which were significantly more common in the OC group (p囚0.001 for both). Demographic, clinical, laboratory findings, indications and results of imaging studies, and antimicrobial treatment of patients are depicted in Table 1. 
Table 1

Demographic, clinical, and laboratory parameters, imaging and treatment associated data of patients with orbital infections

\begin{tabular}{|c|c|c|c|c|}
\hline & $\begin{array}{l}\text { Overall patients } \\
(n=375)\end{array}$ & Preseptal group $(n=340)$ & Orbital/postseptal group $(n=35)$ & $P$ \\
\hline Age, median, months & $44(1-192)$ & $38(1-192)$ & $84(12-168)$ & $凶 0.001$ \\
\hline Gender, boys, n (\%) & $202(53.9 \%)$ & $184(54.1 \%)$ & $18(51.4 \%)$ & 0.761 \\
\hline Male:female ratio & $1.16: 1$ & $1.17: 1$ & $1.05: 1$ & \\
\hline Bilaterally effected eye, n (\%) & $17(4.5 \%)$ & $17(5 \%)$ & 0 & 0.176 \\
\hline Right eye, n (\%) & $191(51 \%)$ & $169(49.7 \%)$ & $22(62.9 \%)$ & 0.235 \\
\hline \multicolumn{5}{|l|}{ Symptoms } \\
\hline Fever & $156(41.6 \%)$ & $128(37.6 \%)$ & $28(80 \%)$ & $凶 0.001$ \\
\hline Periorbital edema & $375(100 \%)$ & $340(100 \%)$ & $35(100 \%)$ & 0.577 \\
\hline Hyperemia & $352(93.9 \%)$ & $318(93.5 \%)$ & $34(97.1 \%)$ & 0.396 \\
\hline Conjunctivitis & $107(28.5 \%)$ & $97(28.5 \%)$ & $10(28.6 \%)$ & 0.996 \\
\hline Eye discharge & $137(36.5 \%)$ & $116(34.1 \%)$ & $11(31.4 \%)$ & 0.746 \\
\hline URTI symptoms & $99(26.4 \%)$ & $81(23.8 \%)$ & $18(51.4 \%)$ & $凶 0.001$ \\
\hline Duration of symptoms, days & $3(1-15)$ & $3(1-15)$ & $5(2-15)$ & $凶 0.001$ \\
\hline Duration of hospitalization, days & $4(1-21)$ & $4(1-14)$ & $8(1-21)$ & $凶 0.001$ \\
\hline \multicolumn{5}{|l|}{ Laboratory evaluations } \\
\hline WBC $\left(x 10^{3} / \mathrm{mm}^{3}\right)$ & $11.6(4.3-40)$ & $11.3(4.3-40)$ & $18.9(6.3-28.9)$ & $凶 0.001$ \\
\hline ANC $\left(\times 10^{3} / \mathrm{mm}^{3}\right)$ & $6.12(0.3-34.9)$ & $5.9(0.3-34.9)$ & $16(2.1-24)$ & 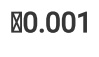 \\
\hline $\operatorname{ALC}\left(\times 10^{3} / \mathrm{mm}^{3}\right)$ & $3.46(0.53-13.6)$ & $3.6(0.53-13.6)$ & $1.8(0.6-8.16)$ & $\mathbb{0 0 . 0 0 1}$ \\
\hline Platelet count $\left(\times 10^{3} / \mathrm{mm}^{3}\right)$ & $342(125-885)$ & $339(125-885)$ & $422(224-789)$ & 0.001 \\
\hline MPV (fL) & $7.9(5.5-11)$ & $8(5.5-11)$ & $7.5(6.2-10.3)$ & 0.004 \\
\hline CRP (mg/dL) & $16(1-450)$ & $13(1-368)$ & $143(10-450)$ & $凶 0.001$ \\
\hline ESR (mm/hour) & $28(2-160)$ & $25(2-95)$ & $76(14-160)$ & $凶 0.001$ \\
\hline NLR & $1.75(0.07-26.6)$ & $1.62(0.07-14.5)$ & $11(1-26.67)$ & $凶 0.001$ \\
\hline PLR & $104(15.3-581.1)$ & $96.9(15.3-581.1)$ & $257(55.2-540)$ & $凶 0.001$ \\
\hline MPVLR & $2.33(0.49-17.74)$ & $2.19(0.49-17.7)$ & $4.1(0.96-17.1)$ & 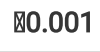 \\
\hline \multicolumn{5}{|l|}{ Imaging and reasons } \\
\hline CT & $57(15.2 \%)$ & $24(7 \%)$ & $33(94.2 \%)$ & $凶 0.001$ \\
\hline MRI & $6(1.6 \%)$ & $1(0.2 \%)$ & $5(14.2 \%)$ & $\otimes 0.001$ \\
\hline Proptosis & $21(5.6 \%)$ & $7(2 \%)$ & $14(40 \%)$ & 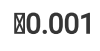 \\
\hline Ophthalmoplegia & $5(1.3 \%)$ & 0 & $5(14.2 \%)$ & $凶 0.001$ \\
\hline Unable to evaluate the eye movements & $37(9.8 \%)$ & $15(4.4 \%)$ & $22(62.8 \%)$ & 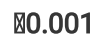 \\
\hline Trauma & $2(0.5 \%)$ & $2(0.5 \%)$ & 0 & 0.221 \\
\hline \multicolumn{5}{|l|}{ Treatment } \\
\hline B lactam / b lactamase inhibitors & $306(81.6 \%)$ & $300(88.2 \%)$ & $6(17.1 \%)$ & 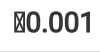 \\
\hline Clindamycin & $55(14.6 \%)$ & $20(5.8 \%)$ & $35(100 \%)$ & $凶 0.001$ \\
\hline 3rd generation cephalosporins & $72(19.2 \%)$ & $43(12.6 \%)$ & $29(82.8 \%)$ & $凶 0.001$ \\
\hline \multicolumn{5}{|c|}{ *All values are given as median (minimum-maximum) } \\
\hline
\end{tabular}




\begin{tabular}{|c|c|c|c|c|}
\hline & $\begin{array}{l}\text { Overall patients } \\
(n=375)\end{array}$ & Preseptal group $(n=340)$ & Orbital/postseptal group $(n=35)$ & $\mathbf{P}$ \\
\hline Glycopeptid & $8(2.1 \%)$ & $3(0.8 \%)$ & $5(14.2 \%)$ & §0.001 \\
\hline Acyclovir & $36(9.6 \%)$ & $36(10.5 \%)$ & 0 & 0.043 \\
\hline \multicolumn{5}{|c|}{ *All values are given as median (minimum-maximum) } \\
\hline
\end{tabular}

Etiology was identified in 312 (83.2\%) patients (Table 2). Overall, the most common etiology was odontogenic infection $(n=71,22.7 \%)$, which was not identified in the OC group $(p=0.003)$.

Table 2

Predisposing factors of all patients

\begin{tabular}{|c|c|c|c|c|}
\hline \multicolumn{2}{|c|}{ Etiology / predisposing factors identified $(n=312)$} & \multirow{2}{*}{$\begin{array}{l}\text { Preseptal group }(n=288) \\
71\end{array}$} & \multirow{2}{*}{$\begin{array}{l}\text { Orbital group }(n=24) \\
0\end{array}$} & \multirow{2}{*}{$\begin{array}{l}p \\
0.003\end{array}$} \\
\hline Odontogenic infections & $71(22.7 \%)$ & & & \\
\hline Conjunctivitis & $64(20.5 \%)$ & 64 & 0 & 0.100 \\
\hline Sinusitis & $46(14.7 \%)$ & 26 & 20 & $\triangle 0.001$ \\
\hline Maxillary & 10 & 5 & 5 & \\
\hline Pansinusitis & 8 & 2 & 6 & 0.533 \\
\hline Maxillary and ethmoid sinusitis & 9 & 2 & 7 & \\
\hline Ethmoid sinusitis & 2 & 0 & 2 & \\
\hline Herpes virus infections & $35(11.2 \%)$ & 35 & 0 & 0.046 \\
\hline Dacryocystitis & $30(9.6 \%)$ & 30 & 0 & 0.067 \\
\hline Nasolacrimal duct obstruction & $12 / 30$ & 12 & 0 & \\
\hline Insect bite & $24(7.6 \%)$ & 24 & 0 & 0.104 \\
\hline Trauma & $14(4.4 \%)$ & 14 & 0 & 0.221 \\
\hline Hordeolum & $10(3.2 \%)$ & 10 & 0 & 0.304 \\
\hline Occult bacteremia & $8(2.5 \%)$ & 8 & 0 & 0.359 \\
\hline Otitis media & $7(2.2 \%)$ & 6 & 1 & 0.649 \\
\hline Idiopathic orbital inflammation & $3(0.9 \%)$ & 0 & 3 & $\varangle 0.001$ \\
\hline
\end{tabular}

Blood culture positivity was not detected in any of the patients. Lumbar puncture and cerebrospinal fluid (CSF) culture was performed in 37 ( $9.9 \%$ ) patients. No microorganism was detected in any of the CSF cultures. The median age of patients who underwent LP was lower than those who did not [3 months (minmax: 1-15 months) vs. 50 months (min-max: 1-192 months), p $邓 0.001$ ]. Conjunctival culture was performed in only 75 (20\%) patients and 40 (53.3\%) of the cultures had no growth. Staphylococcus spp. were the most commonly identified ( $n=18,24 \%)$ organisms in conjunctival cultures (four methicillin-resistant $S$. aureus, 12 methicillin-susceptible $S$. aureus, and two coagulase negative Staphylococci). Streptococcus spp. growth was present in eight (10.6\%) conjunctival cultures (three with S. anginosus group, two with group A Streptococcus, and three with S. viridans). Other microorganisms detected in conjunctival cultures were Streptococcus pneumonia in five, Haemophilus influenza in three and Candida glabrata in one. One conjunctival swab polymerase chain reaction test revealed Adenovirus. 
Three hundred and twenty-eight (87.5\%) patients underwent ophthalmology consultation and 46 (12.3\%) underwent otorhinolaryngology consultation. Sixty (16\%) had undergone orbital imaging (54 had orbital CT and six had orbital MRI). The indications for imaging studies were: inability to evaluate eye movements in 37 (61.6\%) patients, proptosis in 21 (35\%), ophthalmoplegia in four (6.6\%), and history of trauma in five ( $8 \%)$. Thirteen patients (7 boys, 6 girls) with $\mathrm{OC}$ were diagnosed as having subperiosteal/orbital abscess with orbital imaging studies and ten of them needed surgical intervention. Mean abscess diameter was $20.3 \pm 13.6$ millimeters. Median age of the patients with subperiosteal abscess was 120 (min-max: $36-156)$ months and other characteristics are detailed in Table 3. 
Table 3

Demographic and clinical features of children diagnosed with subperiosteal abscess

\begin{tabular}{|c|c|c|c|c|c|c|c|c|c|c|c|}
\hline Patient & $\begin{array}{l}\text { Age } \\
\text { (months) }\end{array}$ & Sex & Symptoms & $\begin{array}{l}\text { Duration } \\
\text { of } \\
\text { symptoms } \\
\text { (days) }\end{array}$ & $\begin{array}{l}\text { Previous } \\
\text { oral } \\
\text { antibiotic } \\
\text { therapy } \\
\text { (days) }\end{array}$ & $\begin{array}{l}\text { Predisposing } \\
\text { factor }\end{array}$ & Treatment & $\begin{array}{l}\text { Method } \\
\text { of } \\
\text { cranial } \\
\text { imaging }\end{array}$ & $\begin{array}{l}\text { Reason for } \\
\text { cranial imaging }\end{array}$ & $\begin{array}{l}\text { Findings on } \\
\text { cranial } \\
\text { imaging }\end{array}$ & Sur \\
\hline 1 & 36 & $\mathrm{~F}$ & $\begin{array}{l}\text { Periorbital } \\
\text { edema } \\
\text { and } \\
\text { hyperemia, } \\
\text { eye } \\
\text { discharge }\end{array}$ & 7 & None & $\begin{array}{l}\text { Not } \\
\text { identified }\end{array}$ & $\begin{array}{l}\text { Ceftriaxone } \\
+ \\
\text { clindamycin }\end{array}$ & CT & $\begin{array}{l}\text { Unable to open } \\
\text { eyelids and } \\
\text { inability to } \\
\text { evaluate the eye } \\
\text { movements }\end{array}$ & $\begin{array}{l}\text { Contrast- } \\
\text { enhancing } \\
\text { subperiosteal } \\
\text { abscess ( } 7 \\
\mathrm{~mm})\end{array}$ & No \\
\hline 2 & 36 & $M$ & $\begin{array}{l}\text { Periorbital } \\
\text { edema } \\
\text { and } \\
\text { hyperemia, } \\
\text { eye } \\
\text { discharge }\end{array}$ & 5 & None & $\begin{array}{l}\text { Maxillary } \\
\text { and ethmoid } \\
\text { sinusitis }\end{array}$ & $\begin{array}{l}\text { Ceftriaxone } \\
+ \\
\text { clindamycin }\end{array}$ & CT & $\begin{array}{l}\text { Unable to open } \\
\text { eyelids and } \\
\text { inability to } \\
\text { evaluate the eye } \\
\text { movements }\end{array}$ & $\begin{array}{l}\text { Peripherally } \\
\text { contrast- } \\
\text { enhancing } \\
\text { abscess } \\
\text { located at } \\
\text { the lateral of } \\
\text { the right orbit } \\
(6 \mathrm{~mm})\end{array}$ & No \\
\hline 3 & 52 & $\mathrm{~F}$ & $\begin{array}{l}\text { Periorbital } \\
\text { edema } \\
\text { and } \\
\text { hyperemia }\end{array}$ & 5 & None & $\begin{array}{l}\text { Not } \\
\text { identified }\end{array}$ & $\begin{array}{l}\text { Ceftriaxone } \\
+ \\
\text { clindamycin }\end{array}$ & CT & Proptosis & $\begin{array}{l}10 \mathrm{~mm} \\
\text { contrast- } \\
\text { enhancing } \\
\text { subperiosteal } \\
\text { abscess }\end{array}$ & Yes \\
\hline 4 & 60 & M & $\begin{array}{l}\text { Fever, } \\
\text { periorbital } \\
\text { edema } \\
\text { and } \\
\text { hyperemia, } \\
\text { eye } \\
\text { discharge }\end{array}$ & 7 & 5 & $\begin{array}{l}\text { Maxillary } \\
\text { sinusitis }\end{array}$ & $\begin{array}{l}\text { Ceftriaxone } \\
+ \\
\text { clindamycin }\end{array}$ & $\mathrm{CT}$ & $\begin{array}{l}\text { Proptosis and } \\
\text { inability to } \\
\text { evaluate the eye } \\
\text { movements } \\
\text { because of } \\
\text { extreme edema }\end{array}$ & $\begin{array}{l}35 \mathrm{~mm} \\
\text { contrast- } \\
\text { enhancing } \\
\text { subperiosteal } \\
\text { abscess } \\
\text { located at } \\
\text { the medial of } \\
\text { the right orbit }\end{array}$ & Yes \\
\hline 5 & 70 & M & $\begin{array}{l}\text { Fever, URTI } \\
\text { symptoms, } \\
\text { vomiting, } \\
\text { periorbital } \\
\text { edema } \\
\text { and } \\
\text { hyperemia }\end{array}$ & 3 & None & $\begin{array}{l}\text { Ethmoid } \\
\text { sinusitis }\end{array}$ & $\begin{array}{l}\text { Ceftriaxone } \\
+ \\
\text { clindamycin }\end{array}$ & CT & Proptosis & $\begin{array}{l}16 \mathrm{~mm} \\
\text { contrast- } \\
\text { enhancing } \\
\text { subperiosteal } \\
\text { abscess near } \\
\text { ethmoid } \\
\text { sinus wall }\end{array}$ & Yes \\
\hline 6 & 76 & M & $\begin{array}{l}\text { Fever, } \\
\text { periorbital } \\
\text { edema } \\
\text { and } \\
\text { hyperemia }\end{array}$ & 7 & 3 & $\begin{array}{l}\text { Not } \\
\text { identified }\end{array}$ & $\begin{array}{l}\text { Ceftriaxone } \\
+ \\
\text { clindamycin }\end{array}$ & MRI & $\begin{array}{l}\text { Ophthalmoplegia } \\
\text { (restricted eye } \\
\text { movements } \\
\text { upwards) }\end{array}$ & $\begin{array}{l}26 \mathrm{~mm} \\
\text { contrast- } \\
\text { enhancing } \\
\text { abscess } \\
\text { displacing } \\
\text { the superior } \\
\text { rectus } \\
\text { muscle }\end{array}$ & Yes \\
\hline 7 & 120 & $\mathrm{~F}$ & $\begin{array}{l}\text { Fever, } \\
\text { periorbital } \\
\text { edema } \\
\text { and } \\
\text { hyperemia, } \\
\text { eye } \\
\text { discharge, } \\
\text { URTI } \\
\text { symptoms }\end{array}$ & 8 & 5 & Pan sinusitis & $\begin{array}{l}\text { Ceftriaxone } \\
+ \\
\text { clindamycin } \\
+ \\
\text { vancomycin }\end{array}$ & CT & $\begin{array}{l}\text { Proptosis and } \\
\text { inability to } \\
\text { evaluate the eye } \\
\text { movements } \\
\text { because of } \\
\text { extreme edema }\end{array}$ & $\begin{array}{l}50 \text { mm } \\
\text { contrast- } \\
\text { enhancing } \\
\text { subperiosteal } \\
\text { abscess in } \\
\text { the medial } \\
\text { wall of right } \\
\text { orbit }\end{array}$ & Yes \\
\hline 8 & 128 & $\mathrm{~F}$ & $\begin{array}{l}\text { Fever, URTI } \\
\text { symptoms, } \\
\text { periorbital } \\
\text { edema } \\
\text { and } \\
\text { hyperemia }\end{array}$ & 4 & None & Pan sinusitis & $\begin{array}{l}\text { Ceftriaxone } \\
+ \\
\text { clindamycin }\end{array}$ & CT & Proptosis & $\begin{array}{l}12 \mathrm{~mm} \\
\text { contrast- } \\
\text { enhancing } \\
\text { abscess in } \\
\text { the left orbit }\end{array}$ & Yes \\
\hline 9 & 132 & $\mathrm{~F}$ & $\begin{array}{l}\text { Periorbital } \\
\text { edema } \\
\text { and } \\
\text { hyperemia }\end{array}$ & 4 & None & Pan sinusitis & $\begin{array}{l}\text { Ceftriaxone } \\
+ \\
\text { clindamycin }\end{array}$ & CT & $\begin{array}{l}\text { Unable to open } \\
\text { eyelids and } \\
\text { inability to } \\
\text { evaluate the eye } \\
\text { movements }\end{array}$ & $\begin{array}{l}40 \mathrm{~mm} \\
\text { contrast- } \\
\text { enhancing } \\
\text { subperiosteal } \\
\text { abscess in } \\
\text { the right orbit }\end{array}$ & Yes \\
\hline
\end{tabular}




\begin{tabular}{|c|c|c|c|c|c|c|c|c|c|c|c|}
\hline Patient & $\begin{array}{l}\text { Age } \\
\text { (months) }\end{array}$ & Sex & Symptoms & $\begin{array}{l}\text { Duration } \\
\text { of } \\
\text { symptoms } \\
\text { (days) }\end{array}$ & $\begin{array}{l}\text { Previous } \\
\text { oral } \\
\text { antibiotic } \\
\text { therapy } \\
\text { (days) }\end{array}$ & $\begin{array}{l}\text { Predisposing } \\
\text { factor }\end{array}$ & Treatment & $\begin{array}{l}\text { Method } \\
\text { of } \\
\text { cranial } \\
\text { imaging }\end{array}$ & $\begin{array}{l}\text { Reason for } \\
\text { cranial imaging }\end{array}$ & $\begin{array}{l}\text { Findings on } \\
\text { cranial } \\
\text { imaging }\end{array}$ & Sur \\
\hline 10 & 144 & $M$ & $\begin{array}{l}\text { Fever, } \\
\text { periorbital } \\
\text { edema } \\
\text { and } \\
\text { hyperemia, } \\
\text { eye } \\
\text { discharge }\end{array}$ & 5 & None & $\begin{array}{l}\text { Maxillary } \\
\text { and ethmoid } \\
\text { sinusitis }\end{array}$ & $\begin{array}{l}\text { Ceftriaxone } \\
+ \\
\text { clindamycin }\end{array}$ & CT & $\begin{array}{l}\text { Proptosis and } \\
\text { inability to } \\
\text { evaluate the eye } \\
\text { movements } \\
\text { because of } \\
\text { extreme edema }\end{array}$ & $\begin{array}{l}40 \text { contrast- } \\
\text { enhancing } \\
\text { orbital } \\
\text { abscess in } \\
\text { the lateral } \\
\text { wall of left } \\
\text { orbit }\end{array}$ & Yes \\
\hline 11 & 144 & $M$ & $\begin{array}{l}\text { Fever, } \\
\text { periorbital } \\
\text { edema } \\
\text { and } \\
\text { hyperemia, } \\
\text { eye } \\
\text { discharge, } \\
\text { URTI } \\
\text { symptoms }\end{array}$ & 8 & 7 & $\begin{array}{l}\text { Maxillary } \\
\text { sinusitis }\end{array}$ & $\begin{array}{l}\text { Ceftriaxone } \\
+ \\
\text { clindamycin }\end{array}$ & CT & Proptosis & $\begin{array}{l}15 \text { orbital } \\
\text { abscess in } \\
\text { the lateral } \\
\text { wall of right } \\
\text { orbit }\end{array}$ & Yes \\
\hline 12 & 156 & $M$ & $\begin{array}{l}\text { Fever, } \\
\text { periorbital } \\
\text { edema } \\
\text { and } \\
\text { hyperemia, } \\
\text { eye } \\
\text { discharge }\end{array}$ & 9 & 7 & $\begin{array}{l}\text { Maxillary } \\
\text { sinusitis }\end{array}$ & $\begin{array}{l}\text { Ceftriaxone } \\
+ \\
\text { clindamycin }\end{array}$ & CT & $\begin{array}{l}\text { Inability to } \\
\text { evaluate the eye } \\
\text { movements } \\
\text { because of } \\
\text { extreme edema }\end{array}$ & $\begin{array}{l}10 \mathrm{~mm} \\
\text { contrast } \\
\text { enhancing } \\
\text { lateral wall } \\
\text { abscess of } \\
\text { orbit }\end{array}$ & No \\
\hline 13 & 156 & $\mathrm{~F}$ & $\begin{array}{l}\text { Fever, } \\
\text { periorbital } \\
\text { edema } \\
\text { and } \\
\text { hyperemia }\end{array}$ & 3 & None & Pan sinusitis & $\begin{array}{l}\text { Ceftriaxone } \\
+ \\
\text { clindamycin }\end{array}$ & MRI & Proptosis & $\begin{array}{l}16 \mathrm{~mm} \\
\text { subperiosteal } \\
\text { abscess } \\
\text { between } \\
\text { frontal bone } \\
\text { and the orbit }\end{array}$ & Yes \\
\hline
\end{tabular}

CT: Computed tomography, F: Female, M: Male, MRI: Magnetic resonance imaging, URTI: Upper respiratory tract infection

Multivariate logistic regression was performed with OC diagnosis as the dependent variable. The results showed that having sinusitis, NLR, symptom duration, platelet count, and proptosis were independently associated with OC diagnosis (Table 4).

Table 4. Factors independently associated with OC diagnosis, multivariable logistic regression

Initial backward conditional regression

(categorical and continuous variables)

$\beta \quad$ Standard Error $p$

coef

Parameters $\quad \beta$

Sinusitis

2.183

NLR

$2.183 \quad 0.876$

$0.385 \quad 0.097$

Platelet count

$0.009 \quad 0.003$

Proptosis

Duration of symptoms (days)

$4.574 \quad 1.132$

$0.662 \quad 0.167$

Dependent Variable: OC diagnosis Backward conditional method. Nagelkerke $\mathrm{R}^{2}=0.790$

NLR: Neutrophil-to-lymphocyte ratio, OC: Orbital cellulitis

Following the identification of these five variables, we derived the OC risk score system (SNIPPED standing for: Sinusitis, NLR, Platelet count, Proptosis, Duration of symptoms) according to beta coefficients (Table 5). Two threshold values were identified from the score: one with respect to the Youden $J$ index of the ROC analysis ( $\geq 7$ points) and the other according to the score at which specificity first reached $100 \%$ ( $\geq 12$ points). With a cut-off value of $\geq 7$, the SNIPPED score had sensitivity, specificity, PPV, NPV, and accuracy values of $91.4 \%, 96.2 \%, 71.1 \%, 99.1 \%$, and \%95.7, respectively.

Table 5. A novel scoring system (SNIPPED score) for predicting orbital cellulitis in pediatric patients 


\begin{tabular}{|c|c|c|c|c|c|}
\hline Parameters & & Weight & & & \\
\hline Sinusitis & If present & 2 points & & & \\
\hline NLR & $>6.78$ & 3 points & & & \\
\hline Platelet count & $>420.5 \times 10^{3} / \mathrm{mm}^{3}$ & 2 points & & & \\
\hline Proptosis & If present & 4 points & & & \\
\hline Duration of symptoms & $\geq 4$ days & 4 points & & & \\
\hline Cut-off points & Sensitivity & Specificity & Accuracy & PPV & NPV \\
\hline$\geq 7$ points & $91.4 \%$ & $96.2 \%$ & $95.7 \%$ & $71.1 \%$ & $99.1 \%$ \\
\hline$\geq 12$ points & $20 \%$ & $100 \%$ & $92.5 \%$ & $100 \%$ & $92.4 \%$ \\
\hline
\end{tabular}

NLR: Neutrophil-to-lymphocyte ratio, NPV: Negative predictive value, PPV: Positive predictive value, SNIPPED: Sinusitis, NLR, Platelet count, Proptosis, Duration of symptoms

\section{Discussion}

Differentiating OC from PC without access to imaging can be very difficult. As such, this study was undertaken with the aim to describe our experience with PC and OC, and to identify easily-accessible parameters that could be used to distinguish OC. The results show that the SNIPPED scoring system, which is comprised of sinusitis, NLR, platelet count, proptosis and duration of symptoms, was a reliable tool that could be used to predict OC.

Most cases of $\mathrm{OC}$ are caused by spread of infection from an adjacent infected sinus, especially ethmoid sinuses. Other much less common sources are spread from the globe (panophthalmitis), the eyelids, the lacrimal sac, infected teeth, and orbital foreign bodies. Finally, although rare, penetrating orbital trauma or skin infection of the face, dental infections, surgery involving ocular or dental regions may cause OC [6-8]. In a study from Turkey that evaluated 36 patients with orbital infections (32 PC and $4 \mathrm{OC}$ ) admitted in a 2-year period, the most common predisposing factor was sinusitis in 16 patients (12 PC and 4 $\mathrm{OC}$ ), and all patients with OC had sinusitis as a predisposing factor [9]. A recently published study reported that $68 \%$ of pediatric PC and OC cases had respiratory tract infection or sinusitis [10]. In a retrospective cross-sectional study from a tertiary ophthalmology hospital including 93 pediatric and adult patients (39 OC and 54 PC), it was reported that sinusitis was the most common etiology in both groups and was significantly more frequent in the OC group [11]. In another retrospective study including 122 pediatric patients with $80.3 \% \mathrm{PC}$ and $19.7 \% \mathrm{OC}$, sinusitis was reported to be associated with $\mathrm{OC}$, whereas dental abscess and trauma were associated with PC [2]. In our study, sinusitis was more common in patients with OC- similar to previous studies. The inflammation around the eyes in PC occurs as a complication of paranasal sinusitis. The pathogenesis is attributable to the venous drainage of the eyelid and surrounding structures. OC occurs when the infection in the sinuses spread to the orbit through the neighborhood or venous spread [6, 12].

Among laboratory parameters, regression analysis only revealed that NLR and platelet count were associated with OC. Although inflammatory parameters are elevated in both PC and OC, the absolute levels of acute phase reactants may be helpful in clinical practice [12]. For instance, higher CRP levels were reported to be associated with post-septal disease in a recent study [10]. NLR is a parameter which is studied in bacterial infections such as neonatal sepsis, urinary tract infection and community acquired pneumonia [13-15]. A recent study which included 243 children with orbital infections, of whom 51 (20.6\%) had OC, found that an NLR value of $>3.14$ could differentiate OC from PC with a sensitivity of $75.5 \%$ and a specificity of $77.4 \%$ [16]. Our threshold of NLR (>6.78) in OC patients seems a bit higher compared to the previous study; but these differences may be due to the well-known variations (based on measurement and devices) in NLR values.

Conjunctival cultures are frequently sterile in children with orbital infections. However, the most commonly identified organisms include Streptococcus spp., Staphylococcus spp., respiratory gram-negative organisms, and anaerobes [17]. In our study, the most common was Staphylococcus spp. (24\%). Similarly, in a 10-year report including 94 patients (67 PC and $27 \mathrm{OC}$ ), the most common pathogen was $S$. aureus (local abscess, eye swab, and blood cultures) in both adult and pediatric patients. The authors also noted that S.aureus and S.pyogenes were the only agents identified in pediatric cases, while S. viridans, Pseudomonas spp., Escherichia coli, H.influenzae, Fusobacterium spp., Peptostreptococcus spp. demonstrated growth in the cultures of the adult group [18]. Another study that evaluated 213 pediatric cases with orbital infections reported that orbital cultures were performed in 54 ( $25 \%$ ) children and the most common pathogen was $S$. aureus [19]. In a previous research including 94 children with OC (in which a pathogen was recovered in $31 \%$ of patients), the most commonly identified pathogen was $S$. anginosus group (15\%), while S.aureus was identified in $9 \%$ of all patients [20]. In our study, only three of the cultures yielded $S$. anginosus group. Therefore, empirical treatment should cover all these pathogens in children with orbital infections [12].

There are a few systematic reviews that focus on the management of pediatric patients with periorbital cellulitis [21, 4]. In children with PC, IV antibiotics should be considered for infants and those with signs of serious systemic infection. Because the meninges are susceptible to inoculation in the infant age group, LP should be performed unless the clinical picture precludes meningitis [12]. In our study group, LP was performed in $9.9 \%$ of patients, but meningitis was not diagnosed in any subjects.

$\mathrm{OC}$ is treated with the administration of IV antibiotics after the patient is hospitalized. If the patient fails to respond to antibiotic treatment within 24 to 48 hours, it is reasonable to perform CT to look for an orbital abscess and/or plan for surgery [7, 21]. Orbital abscess development is associated with various factors, such as being older than 3 years old, having peripheral blood neutrophil count greater than $10.000 / \mathrm{mm}^{3}$, presence of periorbital edema and having received treatment with antibiotics previously [22]. All patients in our study group who needed surgery were above 3 years old and the most identified predisposing factor was sinusitis. The orbital surgery rate in the current study was $28.5 \%$ in the OC group and $2.6 \%$ overall. In a study which evaluated 175 
pediatric cases (36 had OC), 27 patients had subperiosteal abscess and surgery was needed in 31 (1.7\%) of all patients [10]. Another study found the rates of surgical intervention as follows: $29 \%$ overall $(n=93), 48.7 \%$ in the 39 patients with OC and $14.8 \%$ in the 54 patients with PC. The higher rates of surgery may be related to the inclusion of adult patients in this study [11]. It was stated that the proportion of patients requiring surgery increased with age in a study which evaluated 40 children treated for subperiosteal abscess [23]. It may be feasible to suggest that older children and those with sinusitis may benefit from being monitored more carefully about the development of subperiosteal abscess. In our study, surgical intervention was required in all but three of the patients with subperiosteal abscess. In these three patients, abscess diameter was small, fever and other symptoms resolved quickly, response was well to antibiotic treatment, and close monitoring was performed. Ultimately, control imaging studies were normal. Patients who underwent surgery did not attend follow-up visits which presents one of the limitations of this study.

The strength of this study is the large number of cases included over a 13-year period. Another strength is that, to our knowledge, this study is the first pediatric research that has found a novel scoring system to distinguish OC from PC. Previous studies show that imaging is required to differentiate between $\mathrm{PC}$ and OC; however, imaging is expensive and, more importantly, may not be available in resource-limited settings. The value of this scoring system comes from the fact that it drives its results from readily available and/or cheaply measured parameters. Although the $\geq 7$-point cut-off has very good accuracy for the diagnosis of $\mathrm{OC}$, the second cut-off point $(\geq 12)$ was identified to describe a value that could be used for definite OC diagnosis in settings where imaging is not readily-available. In addition, this threshold can be used to quickly identify the severity of a patient when clinical suspicion is insufficient or imaging may be delayed.

There are some limitations to discuss. First, this is a retrospective study and carries all limitations associated with this design (ascertainment bias, selection bias). Second, although the number of patients included is respectable, all data is from only one tertiary center; thus, it may be feasible to perform prospective multicenter studies to better evaluate and confirm this scoring system by performing stratification based on various patient-related characteristics, including sex, age, race, and factors affecting the parameters used for scoring. Finally, it must be noted that we include a temporal parameter (duration of symptoms) in the scoring system. It is evident that judging the utility of this variable in patients who apply before 4 days of symptoms will require further studies. In relation, physicians must be aware of the fact that they must repeat score calculation daily until the 4th day when symptoms continue. However, to conclude, we believe that this score may be helpful to distinguish between PC and OC, since the early and promptly diagnosis and treatment of OC may prevent complications.

\section{List Of Abbreviations}

ALC: Absolute lymphocyte count

ANC: Absolute neutrophil count

CRP: C-reactive protein

CSF: Cerebrospinal fluid

CT: Computerized tomography

ESR: Erythrocyte sedimentation rate

ICD-10: International Classification of Diseases-10

IV: Intravenous

LP: Lumbar puncture

MPV: Mean platelet volume

MPVLR: MPV-to-lymphocyte ratio

MRI: Magnetic resonance imaging

NLR: Neutrophil-to-lymphocyte ratio

NPV: Negative predictive value

OC: Orbital cellulitis

PC: Preseptal cellulitis

PLR: Platelet-to-lymphocyte ratio

PPV: Positive predictive value

ROC: Receiver operating characteristic

SNIPPED: Sinusitis, neutrophil-to-lymphocyte ratio, platelet count, proptosis, duration of symptoms 
URTI: Upper respiratory tract infection

WBC: White blood cell count

\section{Declarations}

Funding: There is no funding source.

Conflicts of interest: The authors declare that they have no conflicts of interest.

Ethics approval: Ethical approval was obtained from the Ethics Committee of our institution (E-20/12-59).

Consent to participate: N/A

Consent for publication: N/A

Availability of data and material: N/A

Code availability: N/A

\section{Authors' Contributions}

Study conception and design: RY, FNO, MP, GT; Literature review: RY, SO, ZSS, AYS, RGC, Acquisition of data: All authors; Analysis and interpretation of data: RY; Drafting of manuscript: RY, GT; Critical revision for important intellectual content: GT, MP, FNO; Final approval of the version to be published: All authors

\section{References}

1. Gonçalves R, Menezes C, Machado R, Ribeiro I, Lemos JA (2016) Periorbital cellulitis in children: Analysis of outcome of intravenous antibiotic therapy. Orbit 35(4):175-180. doi:10.1080/01676830.2016.1176205

2. Santos JC, Pinto S, Ferreira S, Maia C, Alves S, da Silva V (2019) Pediatric preseptal and orbital cellulitis: A 10-year experience. Int J Pediatr Otorhinolaryngol 120:82-88. doi:10.1016/j.ijporl.2019.02.003

3. Gordon AA, Phelps PO (2020) Management of preseptal and orbital cellulitis for the primary care physician. Dis Mon:101044. doi:10.1016/j.disamonth.2020.101044

4. Williams KJ, Allen RC (2019) Paediatric orbital and periorbital infections. Curr Opin Ophthalmol 30(5):349-355. doi:10.1097/icu.0000000000000589

5. Mehta HB, Mehta V, Girman CJ, Adhikari D, Johnson ML (2016) Regression coefficient-based scoring system should be used to assign weights to the risk index. J Clin Epidemiol 79:22-28. doi:10.1016/j.jclinepi.2016.03.031

6. Olitsky S, Marsh JD, Jackson MA (2020) Orbital Infections. In: Kliegman RM., St Geme, JM., Blum, NJ., Shah, SS., Tasker, RC., Wilson, KW. (ed) Nelson Textbook of Pediatrics, 21th Edition. Elsevier, pp 3391-3393

7. Bhatt A (2019) Ocular infections. In: Cherry J.D., Harrison, G.J., Kaplan, S.L., Steinbach, W.J., Hotez, P.J. (ed) Feigin and Cherry's Textbook of Pediatric Infectious Diseases, 8th edition. Elsevier, Philedelphia, pp 581-583

8. McNab A (2019) Orbital Infection and Inflammation. In: Yanoff M., Duker, JS. (ed) Ophthalmology, 5th Edition. Elsevier, pp $1352-1357$

9. Aygün D, Doğan C, Hepokur M, Arslan O, Çokuğraş H, Camcıoglu Y (2017) Evaluation of patients with orbital infections. Turk Pediatri Ars 52(4):221-225. doi:10.5152/TurkPediatriArs.2017.5511

10. Murphy DC, Meghji S, Alfiky M, Bath AP (2020) Paediatric periorbital cellulitis: A 10-year retrospective case series review. J Paediatr Child Health. doi:10.1111/jpc.15179

11. Bagheri A, Tavakoli M, Aletaha M, Salour H, Ghaderpanah M (2012) Orbital and preseptal cellulitis: a 10-year survey of hospitalized patients in a tertiary eye hospital in Iran. Int Ophthalmol 32(4):361-367. doi:10.1007/s10792-012-9574-9

12. Wald ER (2018) Preseptal and Orbital Infections. In: Long SS, Prober CG, Fischer M (eds) Principles and Practice of Pediatric Infectious Diseases, Fifth Edition. Elsevier, Philedephia, pp 517-522

13. Karabulut B, Alatas SO (2021) Diagnostic Value of Neutrophil to Lymphocyte Ratio and Mean Platelet Volume on Early Onset Neonatal Sepsis on Term Neonate. J Pediatr Intensive Care 10(2):143-147. doi:10.1055/s-0040-1715104

14. Han SY, Lee IR, Park SJ, Kim JH, Shin JI (2016) Usefulness of neutrophil-lymphocyte ratio in young children with febrile urinary tract infection. Korean J Pediatr 59(3):139-144. doi:10.3345/kjp.2016.59.3.139

15. de Jager CP, Wever PC, Gemen EF, Kusters R, van Gageldonk-Lafeber AB, van der Poll T, Laheij RJ (2012) The neutrophil-lymphocyte count ratio in patients with community-acquired pneumonia. PLoS One 7(10):e46561. doi:10.1371/journal.pone.0046561

16. Ohana-Sarna-Cahan L, Hurvitz N, Gross I, Cohen A, Hashavya S (2020) Factors Associated With Increased Risk of Pediatric Orbital Cellulitis-Who Should Be Scanned? Pediatr Emerg Care. doi:10.1097/pec.0000000000002083

17. Stimes GT, Girotto JE (2019) Applying Pharmacodynamics and Antimicrobial Stewardship to Pediatric Preseptal and Orbital Cellulitis. Paediatr Drugs 21(6):427-438. doi:10.1007/s40272-019-00357-3

18. Liu IT, Kao SC, Wang AG, Tsai CC, Liang CK, Hsu WM (2006) Preseptal and orbital cellulitis: a 10-year review of hospitalized patients. J Chin Med Assoc 69(9):415-422. doi:10.1016/s1726-4901(09)70284-9

Page $11 / 12$ 
19. Upile NS, Munir N, Leong SC, Swift AC (2012) Who should manage acute periorbital cellulitis in children? Int J Pediatr Otorhinolaryngol 76(8):1073-1077. doi:10.1016/j.ijporl.2012.04.012

20. Seltz LB, Smith J, Durairaj VD, Enzenauer R, Todd J (2011) Microbiology and antibiotic management of orbital cellulitis. Pediatrics 127(3):e566-e572. doi:10.1542/peds.2010-2117

21. Wong SJ, Levi J (2018) Management of pediatric orbital cellulitis: A systematic review. Int J Pediatr Otorhinolaryngol 110:123-129. doi:10.1016/j.ijporl.2018.05.006

22. Rudloe TF, Harper MB, Prabhu SP, Rahbar R, Vanderveen D, Kimia AA (2010) Acute periorbital infections: who needs emergent imaging? Pediatrics 125(4):e719-e726. doi:10.1542/peds.2009-1709

23. Garcia GH, Harris GJ (2000) Criteria for nonsurgical management of subperiosteal abscess of the orbit: analysis of outcomes 1988-1998. Ophthalmology 107(8):1454-1456. doi:10.1016/s0161-6420(00)00242-6 discussion 1457-1458. 